\title{
Das neue Antidiskriminierungsrecht, (k)ein Aufbruch zu neuen Ufern?
}

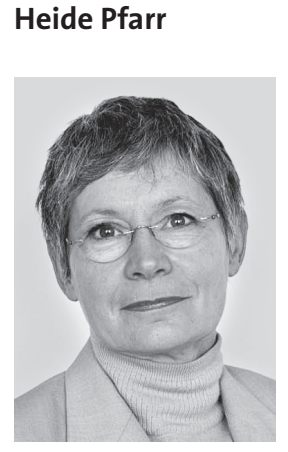

Prof. Dr. Heide Pfarr Wissenschaftliche

Direktorin des

Wirtschafts- und

Sozialwissenschaft-

lichen Instituts in der

Hans-Böckler-Stiftung,

Düsseldorf
Ich könnte mir gut vorstellen, dass Sie eigentlich keine große Lust haben, ein Referat über das Allgemeine Gleichbehandlungsgesetz zu hören. Schließlich gibt es massenhaft dicke Kommentare und Aufsätze zu diesem Thema in den Zeitschriften, die anzuschauen wir professionell verpflichtet sind. Und selbst ein Arbeitgeberanwalt fragt skeptisch, ob all die Fragen zum AGG, die da umfangreich und mehr oder weniger luzide beantwortet werden, sich wirklich stellen und beantwortet werden müssen. Diese Kerbe möchte ich nun nicht vertiefen. Ich gehe nicht einmal im Einzelnen auf die konkreten Regelungen des Gesetzes ein - ein grober Überblick, den Sie ja alle haben, muss genügen. Denn ich möchte die Gelegenheit nutzen, einige grundsätzlichere Fragen im Zusammenhang mit dem AGG zu stellen.

Es sind folgende:

1. Was sagt uns das rechtspolitische Getöse um das AGG?

2. Ist das Gesetz effizient?

3. Was bleibt zu tun?

Die Beratung und Verabschiedung des AGG war von einer aberwitzigen Gegenkampagne der deutschen Wirtschaft begleitet worden. Auch in der Politik waren die schrillen Töne so verbreitet, dass es für dieses Gesetz wohl keine Mehrheit in den Gesetzgebungsorganen der Bundesrepublik gegeben hätte ohne den Druck von Sanktionen aus Europa, gezielt auf die Umsetzung der entsprechenden Richtlinie. Hält man sich vor Augen, welche wüsten Folgen des AGG ausgemalt worden waren, fragt man sich, woher diese Hysterie kam. Von Klageflut war die Rede, von unendlichen Kosten und einer unbeherrschbaren Bürokratie, die auf die Unternehmen, Firmen und Vermieter zukäme. Nur die Bauindustrie würde profitieren, da Millionen Quadratmeter gebaut werden müssten, um all die Akten unterzubringen, die nunmehr zum Beleg ihrer Diskriminierungsfreiheit jahrzehntelang aufbewahrt werden müssten. Hier und nur hier, meine sehr verehrten Damen, liegt offensichtlich der Grund für die Erholung in der Bauwirtschaft, die wir derzeit beobachten.

War es Unkenntnis? Oder was steckte dahinter? Ich bezweifle, dass es bloßer Irrtum war, ge- speist aus der Sorge vor fremdartigen Regelungen. Denn Ansatz wie Reichweite des Gesetzes brachten in dem Rechtsgebiet, das am meisten umstritten war, dem Arbeitsrecht kaum Rechtsänderungen, was die Diskriminierung wegen des Geschlechts betrifft. Mit eher unwichtigen Ausnahmen wiederholt das AGG dazu ja lediglich den Rechtszustand, der schon seit 1980 galt, als das Gleichbehandlungsgebot - gleichfalls auf Druck von Europa - mit den $\mathbb{S} \mathbb{S 6 1 1}$ a und b in das BGB aufgenommen wurde. Dazu sind in den vergangenen 27 Jahren bei juris zum Gleichbehandlungsgebot wegen des Geschlechts 140 Klagen dokumentiert - bei einer geschätzten Diskriminierungsquote über alles von ca. 80 Prozent bei über 30 Millionen Arbeitsverhältnissen. So war offensichtlich, dass es hier auch jetzt nicht zu einer Klageflut kommen konnte.

Nun nimmt dieses Gesetz andere Rechtsgebiete mit auf und dies verbietet weitergehend die Diskriminierung aus Gründen der Rasse oder wegen der ethnischen Herkunft, des Geschlechts, der Religion oder Weltanschauung, einer Behinderung, des Alters oder der sexuellen Identität. Allerdings bezog sich die Heftigkeit der rechtspolitischen Diskussion immer auf das Arbeitsrecht. Und gerade hier konnte man durch minimales Nachdenken prognostizieren, dass heftige, gerichtsförmig ausgetragene Auseinandersetzungen auch zu diesen Diskriminierungsmerkmalen nicht zu erwarten sind. Denn das AGG produziert doch für sie fast dieselben Durchsetzungsschwächen wie für die Umsetzung der $\mathbb{S} 611 \mathrm{a}$ und b BGB. Zur Zeit jedenfalls wird vermeldet, dass es mehr Aufsätze zum AGG gibt als Klagen. Den einzigen Bereich, in der ich eine Rechtsentwicklung über konflikthafte Auseinandersetzungen nicht ausschließen kann, ist die Diskriminierung wegen des Alters dazu werden auch Verfahren genannt. Aber auch da belegt der Blick auf die Rechtsprechung zur Geschlechtsdiskriminierung, dass die Gerichte schon zu vernünftigen Ergebnissen kommen werden - eher allzu verständnisvoll als zu radikal.

All diese Kassandras also wussten genau, dass sie verantwortungslosen Unsinn erzählten - ich nehme die strukturell uninformierte und beratungsresistente Politik hier aus. Das mag ja noch achselzuckend hinzunehmen sein, wenn die Hor- 
rorgemälde nur aus Anwaltsfirmen stammen, die hoffen, verschreckten Arbeitgebern teure Schulungen zu verkaufen, Schulungen mit dem Inhalt: Wie setze ich meine Personalpolitik ungebremst fort, wähle dafür aber eine Form und ein Vorgehen, die das AGG nicht allzu offensichtlich verletzt, eine Personalpolitik, die durchaus auch Frauen und andere Abweichler berücksichtigt, wenn nicht, gerade nicht, noch nicht, nicht mehr oder nicht genügend gesunde deutsche junge Männer für die Besetzung von Arbeitsplätzen zur Verfügung stehen. Gut, das ist widerwärtig, aber Geschäft. Aber warum machen so was BDA und BDI? Und warum beklagten in schöner Breite die Medien die ganz furchtbaren Folgen des AGG?

Nun ist dieses Getöse nichts Einmaliges. Da haben wir gruselige Erfahrungen hinter uns. Denken Sie nur an die Diskussion um die Umsetzung des Gleichberechtigungsartikels in das Familienrecht in den 1950er Jahren. Auch 1980 brach die deutsche Wirtschaft wegen des arbeitsrechtlichen Gleichbehandlungsgebotes wegen des Geschlechts unrettbar zusammen und immerhin meinte damals der Bundesrat in seiner weisen Mehrheit, diskriminieren zu können gehöre doch zu den unveräußerlichen Grundrechten. Ich weiß nicht, ob Ihnen aufgefallen ist, dass hier ein bemerkenswerter Unterschied zu der rechtspolitischen Behandlung anderer Benachteiligter und Förderungsbedürftiger aufgeschienen ist. Die Rechtsgeschichte der Bundesrepublik Deutschland verzeichnet ganz einschneidende Veränderungen in deren rechtliche Lage. Eine ganz wesentliche war etwa, den Schutz und die Ansprüche von Menschen mit Behinderungen nicht davon abhängig zu machen, auf welche Weise ihre Behinderung zustande gekommen ist. So kamen auch jene in den Schutzbereich, die nicht „Kriegsversehrte“ waren. Die Wirtschaft war betroffen durch Abgaben und Verpflichtungen zugunsten von nun erheblich mehr Menschen - aber ein vergleichbares Geschrei wie bei Gesetzgebungsvorhaben zugunsten von Frauen blieb aus. Vielfältige Reformen stellten die Älteren besser - weitgehend im Konsens, ohne Getöse. Insofern werden die unterschiedlichen Benachteiligungsmerkmale, die im AGG zusammengefasst sind, von Wirtschaft und Gesellschaft offensichtlich ganz unterschiedlich wahrgenommen. Gesetz- geberisches Bemühen, hier hilfreich einzugreifen und Diskriminierung abzubauen, wird sehr verschieden bewertet.

Nur Gesetzesreformen zugunsten von Homosexuellen stießen auf ähnlich erbitterten Widerstand in Teilen der Gesellschaft - nicht aber äußerte sich dazu die Wirtschaft negativ wie bei Reformen zugunsten von Frauen. Bemerkenswert!

Die benachteiligten Personengruppen Homosexuelle und Behinderte sind Minderheiten, während die Frauen immerhin die Mehrheit der Bevölkerung ausmachen. Nun gut, es gibt einen weiteren Unterschied: Männer werden mit Sicherheit älter und vielleicht auch krank und zu Behinderten, aber nur in ganz seltenen Ausnahmefällen zu Frauen. (Inzwischen hat sich auch herumgesprochen, dass Männer nicht massenhaft schwul werden, nur weil die Verfolgung und Benachteiligung von Homosexuellen beendet wird.) Insofern können Männer ihre eigenen Interessen verfolgen,

\section{Das Verbot der Diskriminierung von Behinderten, Homosexuellen und Älteren macht diese unsere Gesellschaft gerechter und humaner, aber sie verändert sie nicht in ihrer Struktur.... Die Ungleichbehandlung von Männern und Frauen hingegen ist Ausdruck eines Machtverhältnisses.}

wenn sie Diskriminierungen ablehnen, die auch ihnen drohen können. Diese Erklärung ist zwar unmittelbar plausibel, setzt aber ein Maß skeptischer Wahrnehmung voraus, das gerade dieser Personengruppe eher nicht zuzutrauen ist.

Das Verbot der Diskriminierung von Behinderten, Homosexuellen und Älteren macht diese unsere Gesellschaft gerechter und humaner, aber sie verändert sie nicht in ihrer Struktur. Ein darauf gerichtetes Gesetz muss sich allenfalls den immer beliebteren Vorwurf gefallen lassen, es sei zu sozial. Die Ungleichbehandlung von Männern und Frauen hingegen ist Ausdruck eines Machtverhältnisses. Das Geschlechterverhältnis war immer und ist noch immer durch Macht geprägt. Wer hier rangeht, wer daran etwas Grundsätzliches per Gesetz ändern will, berührt die Machtfrage. Die Reaktion gegenüber einem solchen Angriff ist dann 
nicht rational, nicht abgewogen und argumentativ, da geht es nicht friedlich zu. Denn es wird eine Ordnung bedroht, die nicht nur die unmittelbar Begünstigten schützt, sondern als verfestigte die Welt überschaubar und lebbar macht, ordentlich eben. Sie vermittelt Sicherheit und kann deshalb - und vielfach ist sie es - sogar für jene reizvoll sein, die nicht die Begünstigten dieses Machtverhältnisses sind. Und Ordnung, Sicherheit und Überschaubarkeit sind - jedenfalls für Deutsche - allemal wichtiger als Gerechtigkeit und Gleichheit. Also wird für sie mit harten Bandagen gekämpft, jeder Millimeter des Terrains verteidigt. Das alles natürlich nur in Ausnahmefällen mit zielgerichteter Aggressivität. Nein, absolut guten Gewissens. Man will nur die hergebrachte Ordnung verteidigen, mit der alle gut gelebt haben, doch auch die Frauen, denen es noch nie so gut ging wie heute. Da muss man doch nicht so ein Gewese machen um ein paar Kleinigkeiten und darf nicht gleich alles in Frage stellen.

Die Bekämpfung der Rassendiskriminierung in den USA berührte auch die Machtfrage - die immer auch eine Verteilungsfrage ist. Mir scheint, das ist das Verbot der Diskriminierung wegen Rasse und Ethnie im AGG wohl nicht, jedenfalls solange nicht, wie die Deutschen sich als ganz überwiegende Mehrheit in ihrem Land begreifen und sich für sie hier nicht die Machtfrage stellt.

Also: wäre das AGG als effizientes Gesetz gestaltet, wäre es imstande, sein Versprechen einer diskriminierungsfreien Gesellschaft einzulösen, dann wäre es sozial und gerecht, was die behinderten und die älteren Menschen betrifft. Aber geradezu revolutionär wäre es, wenn es wirklich positiven Einfluss nähme auf das Geschlechterverhältnis. Eine Gesellschaft, die Frauen nicht mehr benachteiligte, eine Gesellschaft, die Geschlechtergerechtigkeit wahr macht, wäre nicht dieselbe wie die, die wir haben. Zwischen den Geschlechtern wäre kein Machtgefälle mehr. Eine Gesellschaft, in der Frauen und Männer gleichberechtigt sind, in der sie die gleichen Chancen haben, wird nicht nur ihr Gesicht ändern, sondern auch viele ihrer Strukturen. Und zwar grundsätzlich ändern, nicht nur in Details. Das geht von der Wirtschafts-, Sozialund Beschäftigungspolitik bis zur Stadtplanung, dem Umweltschutz, das beeinflusst die Bedeutung des öffentlichen Sektors, das wirkt sich aus auf die Unternehmen und ihre Produkte, auf ihre Personalpolitik, die Arbeitsabläufe. Das änderte die Bedeutung von Zeit und Privatheit, ja, auch die Verteilung von Armut und Reichtum.

Und so sind der Widerstand gegen das Gesetz und das Geschrei darum doch gleich viel verständlicher. Wer will denn schon eine Revolution? In Deutschland? Und dann auch noch so eine! Durch Gesetz! Mit Billigung der Regierung! Das muss verhindert werden. Der heftige Widerstand derer, die alles unverändert lassen wollen, ist im Prinzip sehr berechtigt, denn: selbst wenn das Gesetz gar nicht effizient sein sollte - wehret den Anfängen!

Damit bin ich nun schon mitten in meiner zweiten grundsätzlichen Frage: Ist das AGG denn ein effizientes Gesetz? Ein Aufbruch zu neuen Ufern? Also jetzt mal ganz abgesehen davon, dass es mannigfache technische Schwächen hat und in Teilen europarechtswidrig ist. Einfach nur die Frage: Erreicht es diejenigen Ziele, die es vorgibt, erreichen zu wollen? Sie haben sicher Verständnis für diese Formulierung. Ich frage nach dem amtlich benannten Gesetzesziel und um Gottes willen nicht nach dem Ziel des Gesetzgebers. Der wollte nur keinen teuren Krach mit Europa und sonst gar nichts - einige wenige Individuen in Regierung und Bundestag ausgenommen.

Noch mal: das Allgemeine Gleichbehandlungsgesetz soll in öffentlichen und privaten Lebensbereichen systematisch Schutz gegen Diskriminierung aus Gründen der Rasse oder wegen der ethnischen Herkunft, des Geschlechts, der Religion oder Weltanschauung, einer Behinderung, des Alters oder der sexuellen Identität bieten. Anspruchsvolle gesellschaftliche Wertvorstellungen sollen im Zivilrecht durchgesetzt werden, in dem bestimmte Unterschiede und Merkmale, die Personengruppen haben oder die ihnen zugeschrieben werden, bei Verträgen keine Rolle mehr spielen dürfen. Es geht also um das Verhalten jener, die im Markt ihre Vorstellungen typischerweise durchsetzen können. Das soll diskriminierungsfrei werden.

Maßstab für die Effizienz eines solchen Gesetzes, das Verhalten steuern und ändern soll, ist nicht die moralische Schönheit der Deklaration. Es sind vielmehr zwei Kontrollfragen: Welche rechtlichen und tatsächlichen Durchsetzungsmöglichkeiten haben diejenigen, die von dieser Regelung unmittelbar profitieren sollen, und welche Vermeidungs- und Beharrungsmöglichkeiten haben die durch das Gesetz Verpflichteten.

Zweifellos bedeutet es einen Rechtsfortschritt, dass Benachteiligungen wegen der Merkmale Rasse, Alter, Religion, Behinderung nun im Zivilrecht auch ausdrücklich für unzulässig erklärt werden. (Grundrechtliche Schutzpflichten hatten allerdings schon früher in diese Richtung gewiesen). Die Freude darüber entspringt nicht nur der political correctness, sondern auch unserem geschärften Bewusstsein als Betroffene dafür, dass die Zuschreibung von Eigenschaften und Rollen und die Abwertung von „Anderssein“ unerträglich und unwürdig sind. Ich habe aber schon vorhin dargestellt: das Gesetz bringt aber keinen oder nur einen minimalen Rechtsfortschritt im Bereich der Geschlechtsdiskriminierung. Hier handelt es sich um die Wiederholung eines Benachteiligungsverbots, das seit 27 Jahren gilt, und die Gleichstellung der Frauen in der Erwerbsarbeit auch nicht annähernd erreicht hat. Insofern gibt es eine Angleichung der Diskriminierungsverbote, aber eben auf niedrigstem Niveau.

Was bedeutet es eigentlich - mit Blick auf die Effizienz des AGG - für die Frauen, dass ihre Diskriminierung wegen des Geschlechts nun ein Fall unter mehreren ist und als eine Facette möglicher Benachteiligungen aufscheint? Schon bei der Inthronisation von gender mainstreaming wurde die kritische Frage laut: und was ist mit Frauenförderung? Geht die jetzt unter, weil ja alle entsprechend ihrem Geschlecht behandelt und hie und da - sehr hie und da - auch mal 
Männer dabei benachteiligt (und nicht immer nur bevorzugt) werden? Und bei diversity ist ja auch alles umfasst. Nutzt oder schadet dieses Ineinssetzen von unterschiedlichsten Diskriminierungen dem Anspruch der Frauen auf Gleichstellung? Oder profitieren alle - auch die Frauen von dieser Zusammenfassung?

Wir wissen, dass die Gleichsetzung von Geschlechtsdiskriminierung mit der Rassendiskriminierung in den USA den Frauen genutzt hat. Sie ist damals nur aus Zufall in die eigentlich rassisch gerichtete Antidiskriminierungsgesetzgebung mit aufgenommen worden. Und mir scheint, die Gleichsetzung hat in den USA viele Diskussionen, die in unserer Gesellschaft geführt wurden und werden, schon im Ansatz als albern entlarvt. Etwa die Frage: sind Frauen denn dazu überhaupt imstande? - konnte weniger emphatisch verneint werden, wenn die Antwort auch am Maßstab des schwarzen Mannes getroffen werden musste. Offensichtlich geht es danach leichter, vorgeblich sachliche Gründe für eine Ungleichbehandlung zurückzuweisen. Gilt das auch bei uns - als Folge des AGG? Hilft es den Frauen auch bei uns? In Europa, in Deutschland, sind doch die historischen Erfahrungen und Prägungen der Staaten und Gesellschaften mit Diskriminierungen durchaus anders als in den USA. Ich zweifle daran, dass die Zusammenfassung der unterschiedlichen Diskriminierungsmerkmale bei uns der Durchsetzbarkeit nutzt. Mehr noch: Es ist ja nicht ausgeschlossen, dass der Maßstab dadurch nach unten geregelt wird. Sicherlich werden wir auf die Praxis ein kritisches Auge haben müssen.

Das AGG ist eigentlich ein Verbotsgesetz. Es verbietet die Diskriminierung wegen der in ihm benannten Merkmale, darunter das Geschlecht - so, wie Art. 3 Absatz 2 Grundgesetz zunächst auch verstanden wurde und das Arbeitsrechtliche Gleichberechtigungsgesetz des Bundes von 1980 angelegt war. Es verbietet also negative Handlungen und gibt Diskriminierten das Recht, sich individuell zu wehren, es verlangt aber nicht positives Tun zum Zwecke der Gleichstellung. Derartiges lässt es gerade allenfalls mal zu. Das AGG hat die europarechtliche Vorgabe in außerordentlich restriktiver Weise umgesetzt - andere Länder der EU haben erheblich bessere Gesetze. Überhaupt kein Aufbruch zu neuen Ufern.

Auch wir waren schon mal weiter, jedenfalls in der rechtspolitischen Debatte. In der Diskussion um die Verfassungsreform, die dann 1994 umgesetzt wurde und an der sich Mitglieder des djb und er selbst in maßgeblicher Weise beteiligt haben, war wesentliche Erkenntnis, dass ein bloßes Verbot nicht ausreicht, sondern die Durchsetzung der Gleichberechtigung ein förderndes Tun des Staates verlangt. Was aber ist denn seitdem geschehen, außerhalb von Familienpolitik? Mannigfache Gleichstellungsgesetze für den Öffentlichen Dienst sind erlassen worden, darunter auch solche, die aktive Gleichstellungsbemühungen verlangen. Gender Mainstreaming ist ein bindendes, aber offensichtlich nicht oder wenig beachtetes Prinzip für das Handeln der Bundesregierung darüber werden wir noch mehr hören auf diesem Kongress.
Die meisten Frauen arbeiten allerdings in der Privatwirtschaft. Für diesen Bereich ist jedoch nur symbolische Politik gemacht worden. Nicht rechtliche Handlungs- und Förderpflichten wurden gesetzlich festgelegt, sondern unverbindliche Vereinbarungen mit den Verbänden der deutschen Wirtschaft. Die haben erwartungsgemäß nichts gebracht.

So bin ich bei meiner abschließenden 3. Frage: Was bleibt zu tun?

Erlauben Sie, dass ich mich auf den Bereich der Erwerbsarbeit beschränke, wobei mir klar ist, dass dieser zwar sehr wesentlich, aber auch nur ein Ausschnitt im Diskriminierungspanorama ist.

Das AGG hat den grundlegenden Fehler des Arbeitsrechtlichen Gleichbehandlungsgesetzes wiederholt. Das hatte die Durchsetzungsmechanismen rein individualistisch ausgestaltet: die Diskriminierte, und sie allein, sollte ihr Recht gegenüber dem übermächtigen Arbeitgeber durchsetzen, und zwar mit Wirkung nur für sich, selbst wenn der Diskriminierung eine betriebliche Struktur zugrunde lag. So wurden selbstverständlich die Unternehmen nicht auf breiter Ebene zu einer grundsätzlichen Überprüfung ihrer betrieblichen Personalpolitik veranlasst. Vom AGG ist deshalb vehement eine Verstärkung und Kollektivierung der realen Abwehrmöglichkeiten gegen Diskriminierungen erwartet worden. Vergebens. Nur etwas bessere Klagerechte des Betriebsrats und restriktiv formulierte Unterstützungsmöglichkeiten von Verbänden haben schließlich das unwillig betriebene Gesetzgebungsverfahren überlebt.

Eine kluge Antidiskriminierungsgesetzgebung, ein Aufbruch zu neuen Ufern, muss im Ansatz anders vorgehen als das AGG. Sie muss da ansetzen, wo die Benachteiligungen ihren Ursprung haben und die Gleichstellung hergestellt werden könnte. Ich werde Sie jetzt nicht mit den Einzelheiten eines solchen Gesetzesvorhabens behelligen. Nur ganz kurz: Ein effektives Gesetz muss die Betriebe in die Pflicht nehmen und die Akteure, die deren Abläufe prägen. Das sind die Unternehmen selbst und die Betriebsräte - wo es sie gibt. Das sind auch die Gewerkschaften, die entsprechende Tarifverträge anstreben und abschließen könnten.

Diesen betrieblichen Akteuren muss die Verantwortung für die Gleichstellung diskriminierter Personengruppen übertragen werden. Aber sie tun es nicht von allein, wie wir wissen. Deshalb hat der Gesetzgeber eine Aufgabe, die nur er erfüllen kann und endlich erfüllen muss: die betrieblichen Akteure und die Tarifvertragsparteien müssen durch ein Gesetz verpflichtet werden, tätig zu werden. Sie müssen rechtlich dazu gebracht werden, sich den Problemen zuzuwenden, die sie gerne, lange und bisher ungestraft vernachlässigt haben. Aktive Antidiskriminierungspolitik muss zu ihrer Pflichtaufgabe gemacht werden, so wie der Arbeitsschutz ihnen als Aufgabe zugewiesen wurde. Dazu bedarf es möglichst positiver und bei anhaltender Verweigerung wohl auch negativer Anreize - was bedeutet, dass es sich nicht nur um eine arbeitsrechtliche Kodifikation handeln kann. 
Ein Gesetz mit einem solchen Ansatz wäre ein modernes Gesetz, das das AGG in diesem Bereich ergänzen und effektuieren würde. Nicht klassischer Behördenvollzug und obrigkeitliche Kontrolle, sondern eine moderne Steuerung gesellschaftlicher Prozesse durch Recht in Form einer durchsetzbaren Verpflichtung zu selbstverantwortlichem Handeln der Akteure und Akteurinnen. Regulierte Selbstregulierung heißt das Schlagwort, oder: verhandeltes Recht mit gesetzlichen Vorgaben.

Was bleibt zu tun, war meine Frage: Christine Bergmann hat als Frauenministerin in der ersten Legislatur rot-grün ein aktivierendes Gleichstellungsgesetz für die Privatwirtschaft von Juristinnen erarbeiten lassen, die allesamt Mitglieder des djb sind. Der djb selbst hat dieses Gesetzesvorhaben unterstützt. Ministerin Bergmann hat dafür gekämpft und ist an den Alphamännchen in der damaligen Bundesregierung gescheitert. Die Unterstützung des djb ist von anderen nicht energisch genug aufgenommen worden. Dieser Gesetzentwurf bezog sich nur auf das Geschlecht. Nach allem, was ich über die gesellschaftliche Bedeutung des Geschlechterverhältnisses gesagt habe, wäre es durchaus zu rechtfertigen, ein solches allein darauf gerichtetes Gleichstellungsgesetz zu verabschieden. Und immerhin ist nicht ausgeschlossen, dass die Zusammenfassung unterschiedlich Diskriminierter in einem Gesetz dessen Effizienz schadet. Jedoch ist für die praktische Rechtspolitik zu überlegen, ob möglicherweise ein Gleichstellungsgesetz für die Privatwirtschaft, das alle im AGG aufgeführten Berechtigten umfasst, in einem Gesetzgebungsver- fahren bessere Chancen hätte als eines nur zugunsten von Frauen. Betrachte ich den Diskurs im europäischen Rechtsraum und die Diskussion um diversity, nehme ich an, dass vieles für die Zusammenfassung spricht.

Das also bleibt zu tun: Der Einsatz für ein Gleichstellungsgesetz, das das AGG in Bereich des Erwerbslebens ergänzt und effektuiert. Der djb muss sich entscheiden, ob er sich dafür stark machen will. Wenn ja, wäre die Überarbeitung des damaligen Gesetzentwurfs zugunsten der anderen Benachteiligten notwendig.

Aber wer würde ein solches Gesetz denn voranbringen, wer den Aufbruch zu neuen Ufern gestalten? Ich sehe keine Akteure, ja nicht einmal Akteurinnen, die noch mal ernsthaft dieses notwendige Reformvorhaben anpacken würden, nicht in dieser Legislaturperiode und auch nicht in der nächsten. Es gibt dafür keine durchsetzungsstarken Protagonistinnen und Protagonisten in den Parteien und Fraktionen. Das hat die rechtspolitische Debatte um das AGG schmerzlich gezeigt. Und auch muss ich einräumen, für eine Ministerin wäre es wohl erneut ein Himmelfahrtskommando, insbesondere für eine, die schon mehrere Tabuthemen angepackt hat. Der Einfluss der Wirtschaft ist ja seit damals nicht kleiner geworden und die Aufmüpfigkeit und das Drohpotential der Frauen nicht größer. Selbst wenn sich alle, zu deren Gunsten das AGG gilt, zusammenschließen und eine solche Forderung gemeinsam gegenüber dem Gesetzgeber vertreten würden, sehe ich schwarz. Es fehlt uns einfach allen an der angemessenen Militanz. Schade eigentlich.

\section{Gender Mainstreaming als gleichstellungspolitisches Instrument: Verstärkung oder Bedrohung bisheriger Gleichstellungspolitik?}

Karin Tondorf

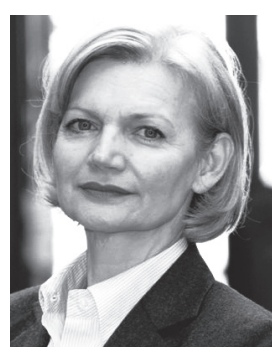

Dr. Karin Tondorf Freiberufliche Wissenschaftlerin und Beraterin, Leiterin des Forschungsund Beratungsbüros GEFA (Gender - Entgelt -Führung-Arbeit), Büro Berlin/Brandenburg
Der Vortrag ist als Powerpoint-Datei auf der djbHomepage abrufbar: http://www.djb.de/Veranstaltungen/Veranstaltungsdokumentationen/20 o7Kongress/

Grundlegend zu Gender Mainstreaming: Gertraude Krell, Ulrich Mückenberger und Karin Tondorf, Gender Mainstreaming: Chancengleichheit (nicht nur) für Politik und Verwaltung, in: Gertraude Krell (Hrsg.) (2008): Chancengleichheit durch Personalpolitik. 5. überarbeitete Auflage, Wiesbaden.

Ausführliche Informationen zu Gender Mainstreaming sind auf den Webseiten des Niedersächsischen Ministeriums für Soziales, Frauen, Familie und Gesundheit zu finden. Um das Ziel der Gleichstellung von Frauen und Männern zu erreichen, verfolgt das Land Niedersachsen eine Strategie des Gender Mainstreaming (GemiNi). Eine Broschüre dazu, hergestellt unter Mitarbeit von Dr. Karin Tondorf, erläutert, was genau sich hinter Gender Mainstreaming verbirgt und welches Potenzial darin steckt. Die Beispiele aus Justiz, Psychiatrie, Steuerverwaltung und Arbeitsmarktpolitik zeigen, wie durch aufmerksames Handeln die Gleichberechtigung von Frauen und Männern ein gutes Stück voran gebracht werden kann. Zudem kann die beschriebene GenderPraxis eines niedersächsischen Landkreises als Vorbild dienen, Gender Mainstreaming erfolgreich auch in den Kommunen umzusetzen.

Niedersächsisches Ministerium für Soziales, Frauen, Familie und Gesundheit (Hrsg.): Gender Mainstreaming in Niedersachsen - Gleiche Chancen, Gleiche Rechte, Politik mit Konsequenz (GemiNi)

http://cdl.niedersachsen.de/blob/images/ C10539984_L2O.pdf (AG) 